Check for updates

Cite this: RSC Adv., 2019, 9, 34880

\title{
Phosphorylation/caproylation of cornstarch to improve its adhesion to PLA and cotton fibers
}

\begin{abstract}
Wei Li, (D)* Zhengqiao Zhang, Jie Wu, Zhenzhen Xu and Zhi Liu
To investigate the influence of phosphorylation/caproylation on the adhesion of cornstarch to polylactic acid (PLA) and cotton fibers for improving its applications, such as in PLA and cotton sizing, herein, a series of phosphorylated and caproylated cornstarch (PCS) samples with different total degrees of substitution (DS) were synthetized by the phosphorylation of acid-converted cornstarch (ACS) with sodium tripolyphosphate (STP) and subsequent caproylation with caproic anhydride (CA). The PCS granules were characterized by Fourier transform infrared spectroscopy and scanning electron microscopy. The adhesion was evaluated by determining the bonding forces of the impregnated PLA and cotton roving. The results of the adhesion measurements were also analyzed, especially for the wetting and spreading of the pastes on the fiber surfaces as well as the failure type and internal stress of the adhesive layers among the fibers. In addition, the viscosity stabilities of the pastes were determined. The results showed that phosphorylation/caproylation was capable of obviously improving the adhesion of starch to PLA and cotton fibers. As the total DS increased, the bonding forces gradually increased. The two substituents improved the wetting and spreading, reduced the internal stress, lowered the layer brittleness, and decreased the probabilities of interfacial failure and cohesive failure, thereby favoring the improvement of the adhesion. The PCS samples with stabilities above $85 \%$ could meet the stability requirement for sizing. Based on the experimental results of the adhesion and the analysis of the results, it can be concluded that PCS shows potential for applications in PLA and cotton sizing.
\end{abstract}

Received 13th September 2019 Accepted 14th October 2019

DOI: $10.1039 / c 9 r a 07384 a$

rsc.li/rsc-advances

\section{Introduction}

Starch is considered as one of the most abundantly occurring glucose polymers. ${ }^{1}$ It includes two polymeric components, known as amylose and amylopectin, and contains numerous $\alpha$ D-glucose units connected through $\alpha-1,4$ linkages and $\alpha-1,6$ linkages. $^{2}$ Moreover, it has especially attractive characteristics such as good biodegradability, ${ }^{3}$ environmental friendliness, low $\operatorname{cost}^{4-6}$ and functional properties; therefore, it is widely used as a sizing agent in the textile field and as a surface-sizing agent in paper making. ${ }^{7,8}$ However, natural starch exhibits low resistance to shear and high temperatures and high tendency towards retrogradation, ${ }^{2}$ leading to fluctuations in the viscosity of cooked starch pastes ${ }^{9,10}$ and subsequently resulting in unstable warp sizing or surface sizing, thereby affecting the quality of the fibers. For this reason, the usability of starch in practical applications is limited. In addition, starch has low waterdispersibility, which adversely affects the wetting and spreading of starch paste on fiber surfaces and produces a negative effect on the adhesion of starch to the fibers.

Undoubtedly, strong adhesion to fibers is perceived to be an essential feature for starch-based products applied for sizing in

College of Textiles and Garments, Anhui Polytechnic University, Wuhu 24100o, Anhui Province, China. E-mail: fangzhiliweiwu@sina.com the textile field and paper making. ${ }^{11}$ During warp sizing, strong adhesion is extremely important; this is mainly because adhesion not only can increase yarn strength, but can also diminish the hairs of warp yarns by gluing them back onto the body of the yarn. ${ }^{12}$ As a result, adhesion has become a significantly important index for evaluating the quality of starch-based products. Fortunately, chemical modification can alter the physicochemical characteristics of native starch, which is usually achieved via derivatization such as esterification, ${ }^{13}$ etherification, ${ }^{14}$ and grafting of starch. ${ }^{15}$ Derivatization can introduce functional substituents into starch molecules to alter their physicochemical properties such as retrogradation behavior and shear resistance. ${ }^{16}$ The alteration of the adhesion behavior provided by substituents introduced via chemical modification has recently received extensive attention.

Polylactic acid (PLA), with its eco-friendly nature, similarity to polyethylene terephthalate and ease of processing, has been extensively explored for fiber applications. ${ }^{17}$ Currently, PLA warps or PLA filaments must be subjected to sizing processes before weaving due to low cohesion, loose tows, and the ready occurrence of entanglement and bonding. Therefore, it is a considerably important task to explore new chemically modified starches for sizing PLA warps or PLA filaments. Obviously, hydrophobic substituents that contain ester groups can increase the van der Waals forces at the interface between 
the starch adhesive layers and PLA fibers due to their chemical similarity to the carbonyls in the PLA chains; thus, they are expected to enhance adhesion. However, an excessive increase in the amount of introduced hydrophobic substituents can lower the water-dispersibility, thereby adversely affecting the wetting and spreading, and decrease the adhesion of the starch to the fibers because the paste adopted in sizing warps is a water-based adhesive. To overcome this shortcoming, an alternative may be the simultaneous introduction of hydrophobic and hydrophilic substituents into the starch molecules. Phosphate and caproate substituents as hydrophilic and hydrophobic substituents, respectively, can be introduced onto starch chains by phosphorylation and caproylation, respectively. The carbonyls in the caproate substituents are chemically similar to the carbonyls in the PLA chains. The hydrophilic phosphate substituents can improve the water-dispersibility of the starch. In addition, phosphate and caproate substituents can disturb the regular arrangement of starch amyloses, partly mitigate the re-association of hydroxyls, and retard retrogradation of the paste during the formation of starch adhesive layers through their strong steric restrictions. Therefore, this restriction $^{\mathbf{1 8}}$ can aid plasticization for the layers, thereby decreasing the internal stresses within the layers. ${ }^{19}$ Therefore, improved adhesion of starch to PLA fibers and hydrophilic cotton fibers can be expected through the simultaneous introduction of hydrophobic caproate and hydrophilic phosphate substituents. In this work, phosphorylated and caproylated cornstarch (PCS) samples were prepared to introduce the phosphate and caproate substituents. The chemical structure of PCS is depicted in Fig. 1.

Currently, no study has investigated phosphorylation together with caproylation as hydrophilic and hydrophobic derivatizations of starch. Therefore, an important objective of this work is to reveal whether phosphate and caproate substituents chemically attached to starch molecules are capable of ameliorating the adhesion of cornstarch to PLA and cotton fibers. Another objective is to confirm the optimal level of starch

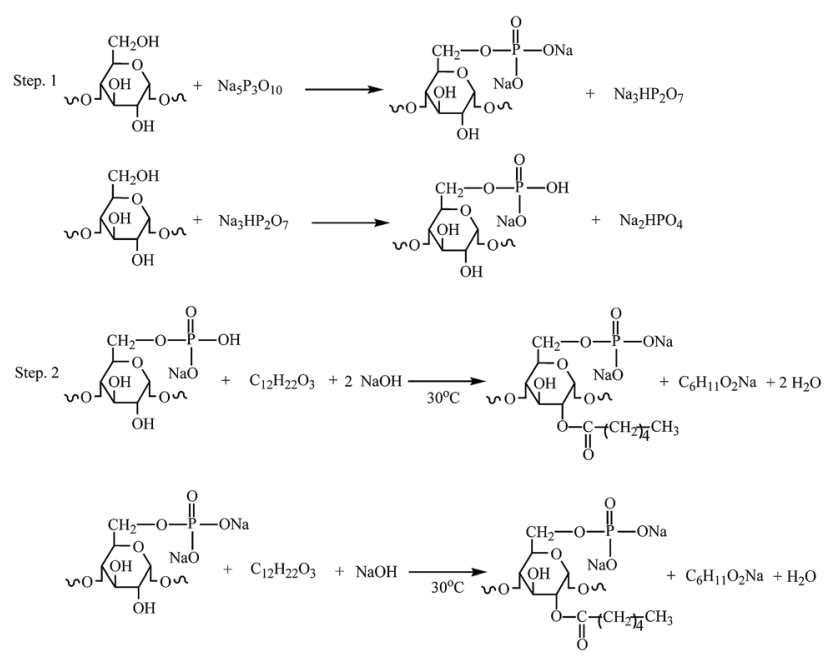

Fig. 1 Two-step reaction for the synthesis of PCS. derivatization for PCS because the functional ability of a modified starch is correlated with its degree of substitution (DS). ${ }^{20}$ The starch samples obtained in this work were characterized using Fourier transform infrared (FTIR) spectroscopy and scanning electron microscopy (SEM). Furthermore, the adhesion of the cooked starch paste to PLA and cotton fibers as well as the light transmittance and the surface tension of the paste were determined; also, film characterization with SEM was performed.

\section{Experimental}

\subsection{Materials and reagents}

Natural cornstarch with an apparent viscosity of $60 \mathrm{mPa} s$ was purchased from Shandong Hengren Industry and Trade Co., Ltd. (Shandong, China). The 100\% cotton roving (460 tex, 112 in twist factor) used in the adhesion measurements was supplied by Talak Cotton Factory (Jiangsu, China). The $100 \%$ polylactic acid (PLA) roving (520 tex) employed for the adhesion measurements was kindly provided by BBCA Group Co., Ltd. (Bengbu, China).

The sodium tripolyphosphate (STP) and caproic anhydride (CA) were supplied by Aladdin Industrial Corporation (Shanghai, China) and were used as received. Other analytically pure reagents, such as hydrochloric acid, sodium hydroxide, anhydrous sodium sulfate and ethanol, were supplied by Sinopharm Chemical Reagent Co., Ltd (Shanghai, China).

\subsection{Synthesis of PCS}

Prior to use, the natural cornstarch was refined with methanoldistilled water medium (85/15 v/v) at $40{ }^{\circ} \mathrm{C}$ (ref. 21) and subsequently acid-converted. ${ }^{22}$ Refinement and acid-conversion were employed to remove the proteins and decrease the viscosity, respectively.

The synthesis of PCS was conducted by a two-step method, i.e., phosphorylation of starch and subsequent caproylation, as shown in Fig. 1. The phosphorylation of starch was conducted by reacting ACS with STP in accordance with the literature. ${ }^{23}$ The caproylation was performed by reacting the phosphorylated starch with CA. The phosphorylated starch was initially mixed and stirred in isopropyl alcohol to form a $40 \%(\mathrm{w} / \mathrm{w})$ dispersion. The dispersion was regulated at $\mathrm{pH} 8.5$ with $2 \%(\mathrm{w} / \mathrm{w}) \mathrm{NaOH}$ aqueous solution and then heated to $30{ }^{\circ} \mathrm{C}$ under continuous agitation. A defined amount of CA was slowly added to the dispersion; meanwhile, the $\mathrm{pH}$ of the dispersion was maintained in the range of 8 to 9 using the $\mathrm{NaOH}$ solution. After the addition, the reaction was conducted at $30{ }^{\circ} \mathrm{C}$ for $0.5 \mathrm{~h}$. Subsequently, the $\mathrm{pH}$ of the dispersion was neutralized to approximately 7 using dilute $\mathrm{HCl}$ solution. The resulting product was vacuum-filtered and washed three times with $75 \%$ ethanol-distilled water. Finally, the starch was oven-dried at $45{ }^{\circ} \mathrm{C}$, ground, and sieved with a 100 -mesh sieve. ${ }^{14}$

\subsection{Characterization of PCS}

A Nicolet Nexus 470 Fourier transform infrared (FTIR) spectrophotometer (Thermo Electron Corporation, Massachusetts, 
USA) was employed to analyze the spectra of the ACS and PCS samples to demonstrate the successful introduction of the substituents on the starch chains. Before the analysis, the ACS and PCS samples were mixed with anhydrous $\mathrm{KBr}$, respectively, and pressed into disk-shaped pellets. The spectra were recorded over the range of 500 to $4000 \mathrm{~cm}^{-1} .^{24}$

The surface morphologies of the PCS and ACS granules and the cross-sections of their films were analyzed with an S-4800 scanning electron microscope (SEM) (Hitachi Limited, Tokyo, Japan). Prior to the analysis, all the samples were sputter-coated with a thin layer of gold.

$\mathrm{DS}_{\mathrm{p}}$ and $\mathrm{DS}_{\mathrm{c}}$ denote the average numbers of hydroxyls substituted by phosphate and caproate substituents per glucose unit, respectively. The phosphorus content $(P \%)$ of the PCS and the $P_{0} \%$ of the ACS were determined at $825 \mathrm{~nm}$ using a UV752N spectrophotometer (Yoke Instruments Co. Ltd., Shanghai, China), respectively. ${ }^{11} \mathrm{DS}_{\mathrm{p}}$ was calculated on the basis of the bonded phosphorus from the results of $P \%$ and $P_{0} \%$ with eqn (1):

$$
\mathrm{DS}_{\mathrm{p}}=\frac{162 \times\left(P-P_{0}\right)}{3100-124 \times\left(P-P_{0}\right)}
$$

where 3100 is the mole mass of phosphorus multiplied by 100 , and 124 is the difference obtained by subtracting the molecular weight of hydrogen from that of the phosphate group.

The caproate content $(C, \%)$ was determined by back titration with $\mathrm{HCl}$ preceded by alkali saponification ${ }^{25}$ and calculated using eqn (2). Also, $\mathrm{DS}_{\mathrm{c}}$ was calculated through eqn (3):

$$
\begin{gathered}
C=\frac{\left(V_{0}-V_{1}\right) \times H \times 0.099}{M} \times 100 \% \\
\mathrm{DS}_{\mathrm{c}}=\frac{162 \times C}{9900-98 \times C}
\end{gathered}
$$

where $V_{0}$ and $V_{1}$ indicate the volumes of $\mathrm{HCl}$ consumed in the blank (ACS was used as the blank sample) and PCS sample titration; $H$ is the molar concentration of $\mathrm{HCl}$ solution; 0.099 denotes the grams in $1 \mathrm{~mL}$ of $1.000 \mathrm{~mol} \mathrm{~L}^{-1} \mathrm{HCl}$ solution; and 9900 and 98 correspond to the molecular weight of caproate $\times$ 100 and the difference between the molecular weights of caproate and hydrogen, respectively.

\subsection{Determination of viscosity stability}

The viscosity stability of the gelatinized starch aqueous paste $(6 \% \mathrm{w} / \mathrm{w})$ was determined using an NDJ-79 Rotary Viscometer (Tongji Electrical Machinery Plant, P. R. China) at a temperature of $95{ }^{\circ} \mathrm{C}$ under a shear rate of $2028 \mathrm{~s}^{-1} \cdot{ }^{25}$ The data reported are the averages of two separate results.

\subsection{Determination of adhesion}

The slightly sized roving method, which has become a standard method (FZ/T 15 001-2008) in China to estimate adhesion, was employed to study the adhesion of the starch samples to the fibers. PCS or ACS (used as a control) was suspended in distilled water to form a $1 \%(\mathrm{w} / \mathrm{w})$ suspension and then completely gelatinized at $95{ }^{\circ} \mathrm{C}$ for $1 \mathrm{~h}$ under subsequent stirring. Afterwards, the paste was transferred into a metal box placed in a water bath at $95{ }^{\circ} \mathrm{C}$. A roving was carefully wound onto a steel frame and immersed into the paste for $5 \mathrm{~min}$; then, the immersed roving was dried in air. The dried roving was subjected to a tensile test to failure, and the bonding force was employed to indicate the adhesion of PCS or ACS to the fibers. Undoubtedly, a high force implies great adhesion to the fibers. Before the tensile tests, the dried roving was equilibrated at $65 \%$ relative humidity and $20{ }^{\circ} \mathrm{C}$ for at least $24 \mathrm{~h}$. The test was determined on a YG065H Tensile Tester (Laizhou Instrument Co. Ltd., Shandong, China) with an initial jaw separation distance of $100 \mathrm{~mm}$ at

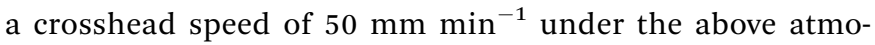
spheric conditions according to a previous publication. ${ }^{26}$ The data were expressed as the averages and standard deviations (SD) of 20 sample tests, among which abnormal values were rejected.

\subsection{Determination of surface tension}

A DCAT 21 Automatic Tensiometer (Dataphysics Co., Ltd., German) was used to determine the surface tension of the cooked starch aqueous paste $(1 \% \mathrm{w} / \mathrm{w})$. The paste was formed by heating a $1 \%$ starch aqueous dispersion to $95{ }^{\circ} \mathrm{C}$ and subsequently maintaining this temperature for $1 \mathrm{~h}$. After the paste was cooled, its tension was determined in duplicate at room temperature.

\subsection{Determination of light transmittance}

The light transmittance of the cooked PCS or ACS paste was measured with Craig's method. ${ }^{27}$ Briefly, a granular starch sample $(1 \mathrm{~g})$ was dispersed in $99 \mathrm{~mL}$ of distilled water and then pasted by heating the dispersion to $95{ }^{\circ} \mathrm{C}$ under mechanical agitation for $1 \mathrm{~h}$. After the obtained paste was cooled to room temperature, the percent transmittance at $640 \mathrm{~nm}$ (ref. 28) was determined immediately using a UV9600 spectrophotometer (Ruili Analytical Instrument Co., Ltd., Beijing, China) against a distilled water blank. The transmittance was determined in duplicate and the average was reported.

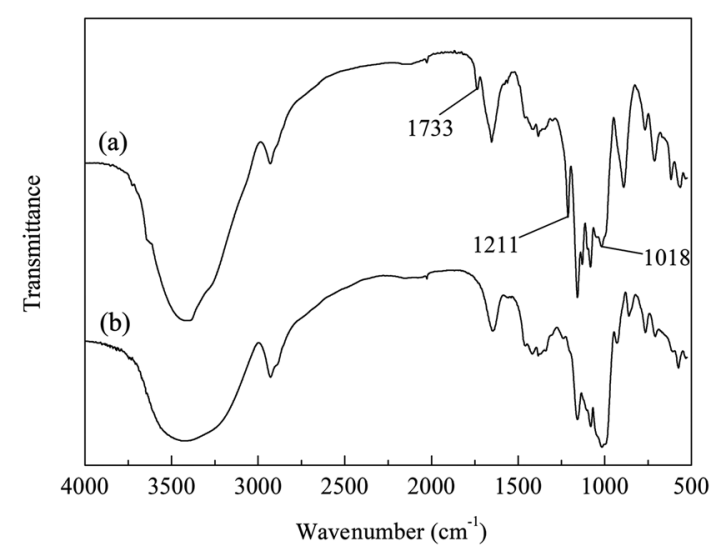

Fig. 2 FTIR spectra of PCS (a) and ACS (b). 


\section{Results and discussion}

\subsection{Characterization}

Fig. 2 depicts the FTIR spectra of PCS (Fig. 2(a)) and ACS (Fig. 2(b)) in the region of 500 to $4000 \mathrm{~cm}^{-1}$. The spectra were adopted to demonstrate the successful introduction of phosphate and caproate substituents on the starch chains. Compared with the characteristic absorption peaks of ACS, a new absorption peak was observed at the wavenumber of $1733 \mathrm{~cm}^{-1}$ in the spectrum of PCS; this was ascribed to the characteristic absorption band of $\mathrm{C}=\mathrm{O}^{24}$ in caproate substituents, proving the successful introduction of caproate substituents into the starch molecules. In addition, two new peaks appearing at $1211 \mathrm{~cm}^{-1}$ and $1018 \mathrm{~cm}^{-1}$ in the spectrum of PCS were assigned to $\mathrm{P}=\mathrm{O}^{29}$ and $\mathrm{C}-\mathrm{O}-\mathrm{P},{ }^{30}$ respectively; this indicates the presence of phosphate substituents on the starch chains.

The DS value denotes the average number of hydroxyls per anhydroglucose unit substituted by phosphate and caproate substituents. In this work, all PCS samples showed a $\operatorname{DS}_{\mathrm{p}}$ (i.e., the phosphorylation extent) of 0.013 , corresponding to $6 \mathrm{wt} \%$ of STP to ACS in the reaction system. The $\mathrm{DS}_{\mathrm{c}}$ and total DS
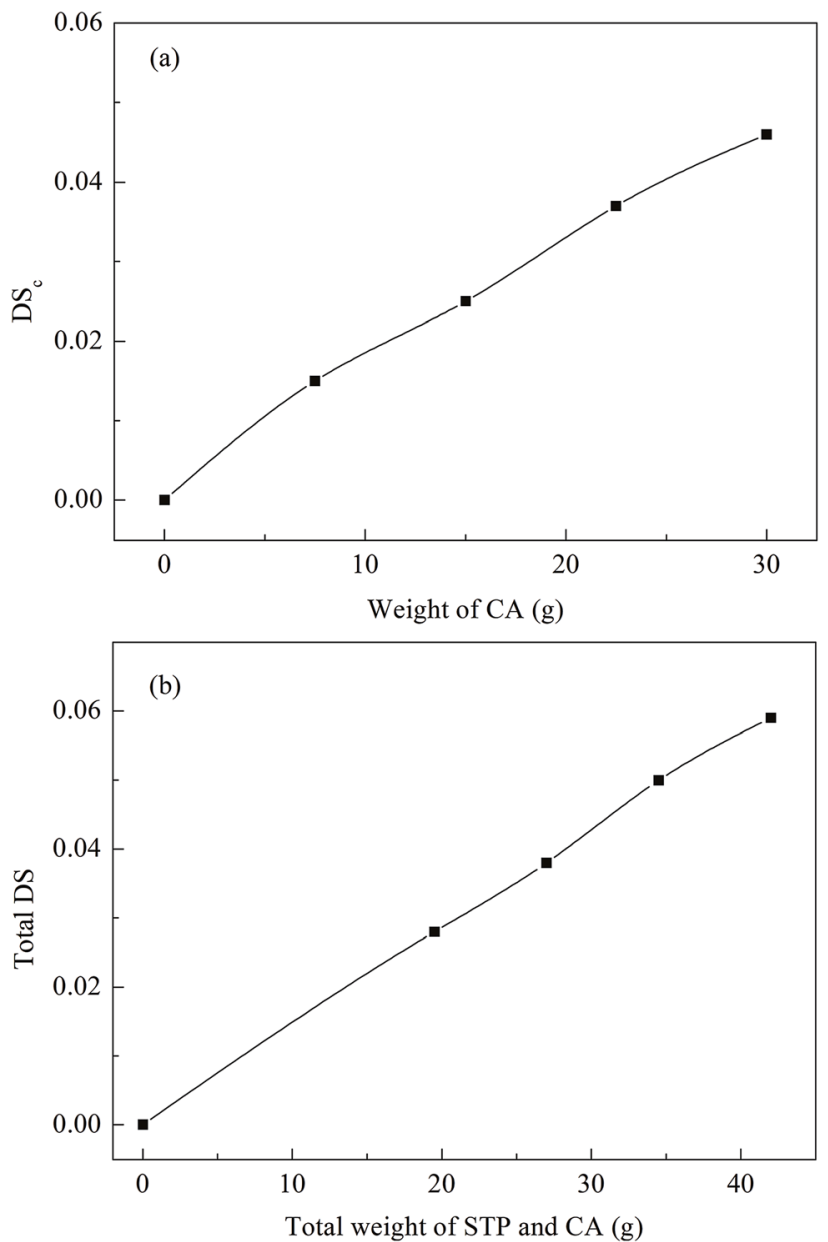

Fig. 3 Characterization of the caproylation extent (a) and total extents of phosphorylation/caproylation (b) of starch. (containing $\mathrm{DS}_{\mathrm{p}}$ and $\mathrm{DS}_{\mathrm{c}}$ ) of the granular PCS samples were also determined, as depicted in Fig. 3(a) and (b), respectively. It can be found from Fig. 3(a) that the $\mathrm{DS}_{\mathrm{c}}$ values (indicating the DS of the caproate substituents) depended on the amounts of CA relative to starch in the reaction system. Also, with increasing amount, the value gradually increased from 0 to 0.046 . Therefore, due to the fixed $\mathrm{DS}_{\mathrm{p}}$ for PCS, the total DS (the sum of $\mathrm{DS}_{\mathrm{p}}$ and $\mathrm{DS}_{\mathrm{c}}$ ) also gradually increased from 0 to 0.059 with an increase in the amount of CA, as shown in Fig. 3(b).

Starch derivatization can involve physicochemical phenomena on the surfaces of the contacting phases. ${ }^{31}$ The SEM technique is an important means of clarifying the granular structures of derivatized starches and the most substituted regions on the starch granules. ${ }^{14}$ SEM images of the starch granules were recorded with the SEM equipment, as represented in Fig. 4, to illustrate the influence of phosphorylation/ caproylation on the surface morphologies of the starch granules. It can be observed from Fig. 4(a) that there were no visible fissures on the surface of the ACS granules. The SEM image of the PCS granules (Fig. 4(b)) shows visible damage on the surface of the PCS granules. When the reaction temperature is lower than the gelatinization temperature of the starch, phosphorylation and caproylation of granular ACS will mainly occur on the
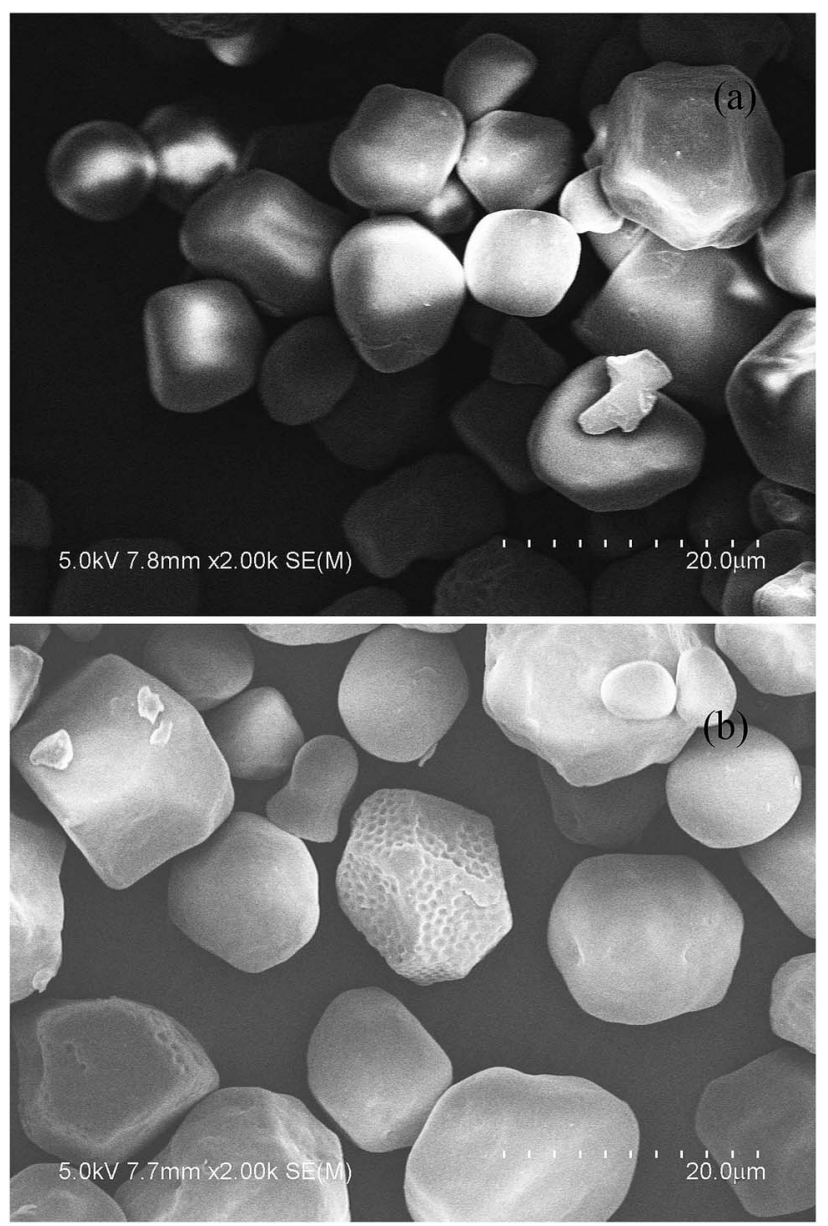

Fig. 4 SEM images of the prepared ACS (a) and PCS (b). 
surfaces of the granules under alkaline conditions. Moreover, alkaline conditions will probably result in damage on the surfaces of the starch granules. As the reaction continues and damage occurs, a portion of the reaction will occur in the interior of the granules with the penetration of the reagents.

\subsection{Influence of phosphorylation/caproylation on the viscosity stability}

The influence of the phosphate and caproate substituents introduced onto the ACS chains on the viscosity stability of the cooked ACS paste is shown in Fig. 5. It can be seen that the PCS samples were superior to their ACS counterparts in the stability of the paste viscosity. As the total DS increased, the stability exhibited a slight decreasing tendency. This indicates that the introduction of caproate substituents has a negative impact on the stability. Fortunately, the stability was above $85 \%$ in the total DS range. Commonly, the required viscosity stability of a paste used in sizing is $\geq 85 \% .^{32}$ As a result, the introduction of phosphate and caproate substituents can meet the stability requirement for warp sizing.

The viscosity of the cooked starch paste must be stable in warp sizing; this can ensure stability of the size add-on, ${ }^{\mathbf{1 4}}$ thereby enhancing the weaving efficiency. Undoubtedly, the $\mathrm{D}^{-}$ glucosidic bonds in starch molecules are sensitive to heat and shear; the bonds will break and degrade the starch chains in the cooked aqueous paste, eventually generating fluctuations in the viscosity ${ }^{33}$ and causing poor stability. Accordingly, the ACS paste has a stability of $83.3 \%$. The introduction of hydrophobic caproates may decrease the stability; this is mainly attributed to the fact that the hydrophobic caproates decrease the interaction and affinity between starch molecules and water, which is likely to increase the extent of degradation. However, the introduction of hydrophilic phosphate substituents can promote the interaction and affinity between starch molecules and water, thus lowering the degradation. Also, the hindrance of the phosphate substituents obstructs the hydrogen bonding between the starch hydroxyls and alleviates the aging of the paste. Accordingly, the phosphate substituents favor improvement in the stability. Fortunately, the combination of the positive effect of

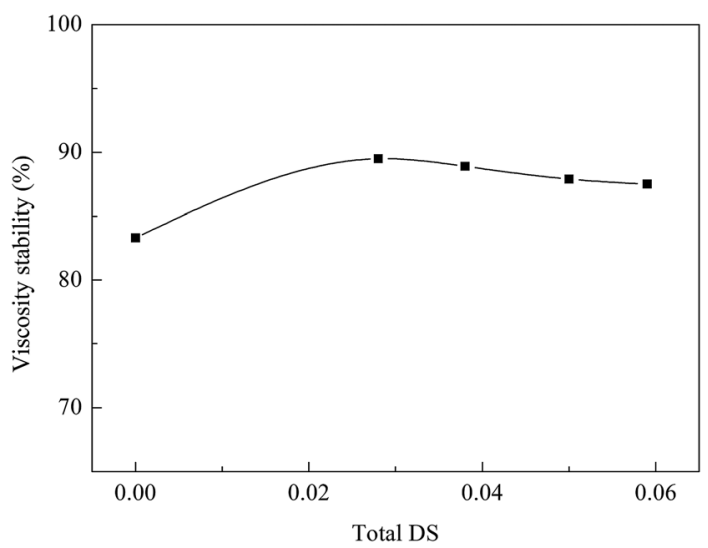

Fig. 5 Influence of phosphorylation/caproylation on the viscosity stability of the cooked starch paste. the phosphate substituents and the negative effect of the caproates resulted in stabilities above $85 \%$ of the prepared PCS samples, thereby ensuring stability of the size add-on during warp sizing.

\subsection{Influence on adhesion}

The influence of phosphorylation/caproylation on the adhesion of starch to PLA and cotton fibers was investigated as a function of the total DS, as represented in Table 1 and 2, respectively. As observed, PCS was superior to ACS (total DS $=0$ ) in its bonding forces to cotton and PLA fibers. The bonding forces of PCS to both fibers depended on the total DS. As the DS increased from 0 to 0.059 , the forces gradually increased from $52.4 \mathrm{~N}$ to $62.2 \mathrm{~N}$ for PLA fibers and from $65.4 \mathrm{~N}$ to $73.1 \mathrm{~N}$ for cotton fibers. These observations imply that phosphorylation/caproylation improves the adhesion of starch to both fibers, and the improvement was gradually enhanced as the total DS increased. Moreover, it could be seen that the increment in the bonding forces of PCS to PLA fibers is higher than that to cotton fibers after the modification.

According to the fracture location, the fracture of an adhesive joint generally involves cohesive and interfacial failure. ${ }^{34}$ The former and latter are failures that occur wholly within the matrix of an adhesive layer formed by the paste covering the fiber surfaces; these failures occur exactly at the interfaces between the layers and fibers, respectively. Obviously, the bonding forces of an adhesive joint can be estimated by its layer properties and interfacial attraction because the fracture of a joint commonly occurs at its weakest area. To study the influences of chemical derivatization on the adhesion, the primary investigation will be of the interfacial interactions at the interfaces, followed by the properties of the layers.

Natural starch inherently appears as insoluble semicrystalline granules and includes two polymeric components (linear amylose and branch amylopectin). ${ }^{35}$ During the drying of starch aqueous paste covering the fiber surfaces, the amyloses are inclined to regularly arrange themselves and closely approach each other by association of the hydroxyls, thereby forming amylose bundles at the supramolecular level. Moreover, amylose molecules are also capable of co-crystallizing with the linear branches of amylopectin ${ }^{36}$ due to this association. ${ }^{37}$ As a result, paste retrogradation of the starch will occur. Due to retrogradation, the starch paste can become microheterogeneous, inevitably inducing incomplete wetting and outspreading of the paste on fiber surfaces. ${ }^{24}$ It has been

Table 1 Influence of phosphorylation/caproylation on the adhesion of starch to PLA fibers

\begin{tabular}{|c|c|c|c|c|c|}
\hline Starch sample & $\mathrm{DS}_{\mathrm{p}}$ & $\mathrm{DS}_{\mathrm{c}}$ & Total DS & $\begin{array}{l}\text { Bonding forces } \\
\text { (N) }\end{array}$ & SD \\
\hline ACS & 0.000 & 0.000 & 0.000 & 52.4 & 3.96 \\
\hline \multirow[t]{4}{*}{ PCS } & 0.013 & 0.015 & 0.028 & 57.8 & 3.86 \\
\hline & 0.013 & 0.025 & 0.038 & 59.4 & 3.83 \\
\hline & 0.013 & 0.037 & 0.050 & 60.4 & 4.25 \\
\hline & 0.013 & 0.046 & 0.059 & 62.2 & 3.59 \\
\hline
\end{tabular}


Table 2 Influence of phosphorylation/caproylation on the adhesion of starch to cotton fibers

\begin{tabular}{llllll}
\hline & & & & \multicolumn{2}{l}{ Bonding forces } \\
Starch sample & $\mathrm{DS}_{\mathrm{p}}$ & $\mathrm{DS}_{\mathrm{c}}$ & Total DS & $(\mathrm{N})$ & $\mathrm{SD}$ \\
\hline ACS & 0.000 & 0.000 & 0.000 & 65.4 & 4.41 \\
PCS & 0.013 & 0.015 & 0.028 & 70.8 & 4.33 \\
& 0.013 & 0.025 & 0.038 & 71.5 & 4.21 \\
& 0.013 & 0.037 & 0.050 & 72.8 & 4.12 \\
& 0.013 & 0.046 & 0.059 & 73.1 & 4.45
\end{tabular}

clarified that incomplete wetting and outspreading are deleterious to adhesion ${ }^{33}$ due to the occurrence of interfacial failure and high stresses around the unwetted or outspread areas. Furthermore, the starch aqueous paste will shrink in volume due to the loss of water during drying, converting into starch adhesive layers between the fibers in the roving. Due to the brittleness of the starch, the shrinkage will induce high internal stresses at the adhesive layer-fiber interfaces and within the bulk phases of the layers. ${ }^{24}$ Local interfacial failure will occur once the local stress is greater than the local strength. Therefore, high stresses can cause intensive damage to the adhesion. ${ }^{38}$

Obviously, the introduced substituents can disturb the association of the hydroxyls and diminish the regular arrangement of the amyloses through steric hindrance, thereby favoring alleviation of retrogradation. ${ }^{14}$ For this reason, improvement in the wetting and spreading may be expected. Additionally, it is broadly accepted that the wetting and spreading abilities of an adhesive liquid onto a given solid surface are closely related to the surface tension of the liquid. ${ }^{24}$ A low tension commonly induces ameliorated adhesion due to improved wetting and spreading. ${ }^{39}$ Therefore, the influences of the phosphate and caproate substituents on the surface tension of cooked starch paste were investigated and are depicted in Fig. 6. As observed, the tension decreased after the introduction of the substituents compared with that of the ACS (total DS $=0$ ) paste. This suggested that the phosphate and caproate

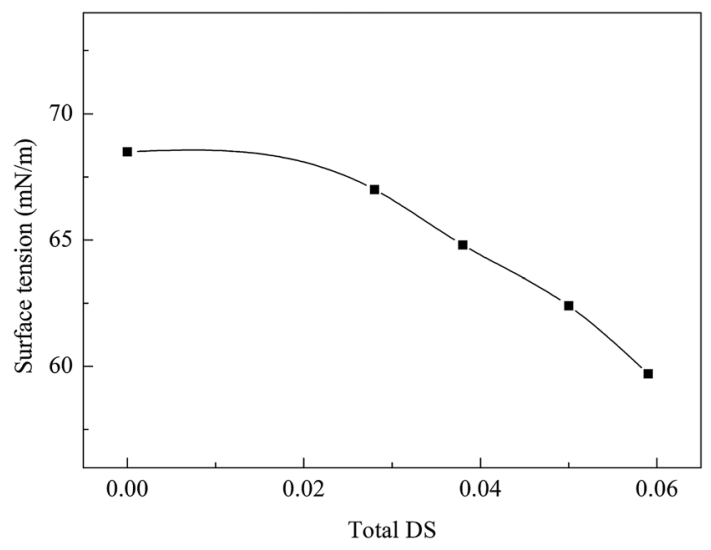

Fig. 6 Influence of the phosphate and caproate substituents on the surface tension of the cooked starch paste. substituents were both necessary to provide derivatives with greater surface activity. The tension was correlated with the total DS, and it gradually decreased in the DS range of 0.028 to 0.059. A gradually decreased tension favors improved wetting and spreading of cooked starch pastes onto fiber surfaces. The improvement will decrease the likelihood of interfacial failure, thereby enhancing the adhesion. Apparently, the hindrance of phosphate and caproate substituents can exhibit strong obstruction of the association and regular arrangements, thereby providing internal plasticization to decrease the stresses at the adhesive layer-fiber interfaces. These factors reinforce the interfacial actions and provide a positive effect on the adhesion. However, the hydrophobic caproate substituents will decrease the water-dispersibility of starch, and the waterdispersibility was estimated by the determination of the light transmittance of the paste, as represented in Fig. 7. As found, with the increase from 0.028 to 0.059 of the total DS (from 0 to 0.046 of the $\mathrm{DS}_{\mathrm{c}}$ ), the transmittances of the PCS pastes gradually decreased. In general, the lower the transmittance, the poorer the water-dispersibility. ${ }^{40}$ Accordingly, with increasing amount of caproate substituents, the water-dispersibility of the PCS paste continuously decreases. It has been concluded that worse water-dispersibility easily produces incomplete wetting and outspreading, ${ }^{13}$ leading to interfacial failure and generating an negative effect on the adhesion. Fortunately, the combination of positive and negative factors may decrease the probability of interfacial failure, thereby favoring adhesion.

In addition, the two substituents demonstrate toughening effects on the layers, which can lower the brittleness of starch adhesive layers and decrease the possibility of cohesive failure. The decrease of the brittleness can be verified by observing the SEM pictures of cross-sections of the ACS and PCS films, as illustrated in Fig. 8. It can be found from Fig. 8(a) that the ACS film exhibited strong brittleness; meanwhile, the PCS film displayed lower brittleness, as can observed from Fig. 8(b). This observation indicates that the two substituents exert toughening effects on the film. Undoubtedly, the PCS film (described as PCS adhesive layers that formed among the fibers) with lower brittleness is preferable to the brittle ACS film to decrease the cohesive failure and improve the adhesion, thereby promoting the applications of PCS in warp sizing.

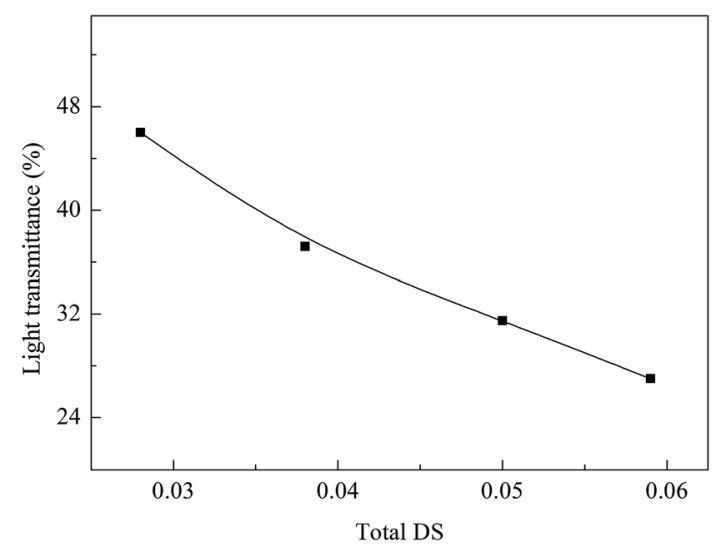

Fig. 7 Light transmittances of the cooked PCS pastes. 

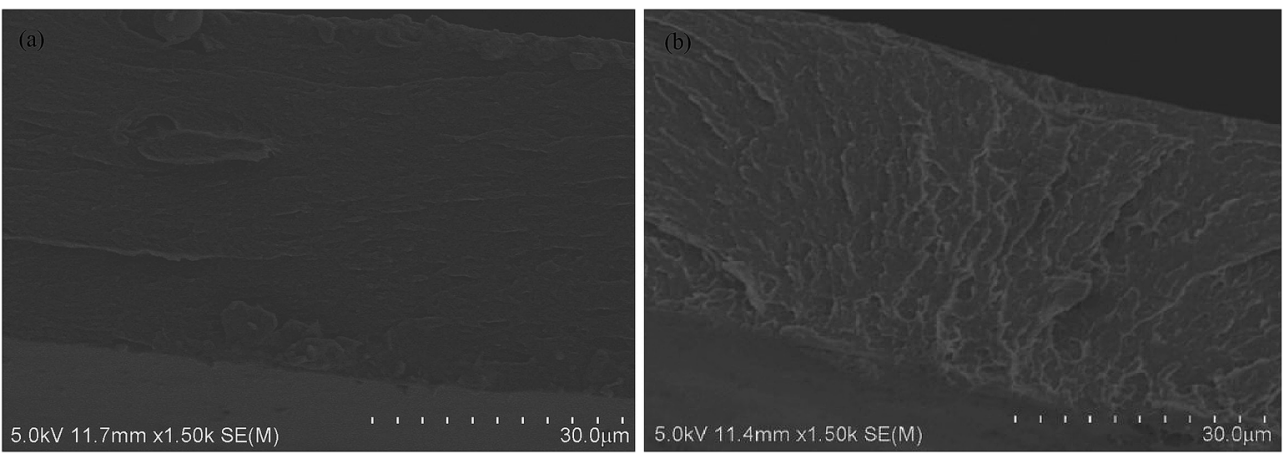

Fig. 8 SEM images of the cross-sections of the ACS (a) and PCS (b) films.

\section{Conclusions}

Based on our results, the incorporation of phosphate and caproate substituents into starch molecules through phosphorylation of ACS with STP and subsequent caproylation with CA is an effective method to ameliorate the adhesion of starch to PLA and cotton fibers and substantially improve its end-use ability in warp sizing. The bonding forces of PCS (total DS $=0.028$ to 0.059) to PLA and cotton fibers were superior to those of ACS $(\mathrm{DS}=0)$; this indicates that phosphorylation/caproylation was able to improve the adhesion of starch to PLA and cotton fibers. The adhesion of PCS to PLA and cotton fibers depended on the total DS. Also, with increasing total DS, the bonding forces gradually increased. The substituents not only alleviate retrogradation via obstructing the re-association of hydroxyls and decreasing the regular arrangement of amylose molecules but also lower the surface tension of the cooked starch paste; this favors improvements in the wetting and spreading and lowers the probability of interfacial failure. Additionally, the two substituents generated a toughening effect on the layers (which was verified by observing the SEM pictures of cross-sections of the ACS and PCS films) and thus decreased the internal stresses within the matrix of the adhesive layers, thereby diminishing the probability of cohesive failure and favoring the adhesion. For these reasons, the derivatization improved the adhesion. Moreover, all the PCS samples had stabilities above $85 \%$; this implies that the introduction of two substituents can meet the stability requirement for warp sizing. Considering the results of PCS regarding the paste stability, adhesion, and preparation cost, PCS with a total DS range of 0.038 to 0.05 shows potential in applications of PLA and cotton sizing.

\section{Conflicts of interest}

There are no conflicts to declare.

\section{Abbreviations}

PCS

DS

ACS
Phosphorylated and caproylated cornstarch

Degree of substitution

Acid-converted cornstarch
STP Sodium tripolyphosphate

CA Caproic anhydride

PLA Polylactic acid

FTIR Fourier transform infrared

SEM Scanning electron microscopes

\section{Acknowledgements}

This work was financially supported by the Key Research and Development Project of Anhui Province (No. 201904a06020001), Natural Science Foundation of Anhui Province (No. 1908085ME124), Pre-research Project of National Natural Science Foundation of China (No. 2018yyzr08), Natural Science Research Project of Universities of Anhui (No. KJ2018ZD012), and Science and Technology Planning Project of Wuhu City (No. 2018pt04), China.

\section{References}

1 B. Ramaraj, Crosslinked poly(vinyl alcohol) and starch composite films. II. Physicomechanical, thermal properties and swelling studies, J. Appl. Polym. Sci., 2007, 103, 909-916.

2 J. Waterschoot, S. V. Gomand, E. Fierens and J. A. Delcour, Starch blends and their physicochemical properties, Starch/ Staerke, 2015, 67, 1-13.

3 A. Cano, E. Fortunati, M. Cháfer, J. M. Kenny, A. Chiralt and C. González-Martínez, Properties and ageing behaviour of pea starch films as affected by blend with poly(vinyl alcohol), Food Hydrocolloids, 2015, 48, 84-93.

4 J. Chen, C. Liu, Y. Chen, Y. Chen and P. R. Chang, Comparative study on the films of poly(vinyl alcohol)/pea starch nanocrystals and poly(vinyl alcohol)/native pea starch, Carbohydr. Polym., 2008, 73, 8-17.

5 J. Y. Xu, E. F. Krietemeyer, V. L. Finkenstadt, D. Solaiman, R. D. Ashby and R. A. Garcia, Preparation of starch-polyglutamic acid graft copolymers by microwave irradiation and the characterization of their properties, Carbohydr. Polym., 2016, 140, 233-237.

6 K. H. Yao, J. Cai, M. Liu, Y. Yu, H. G. Xiong, S. W. Tang and S. Y. Ding, Structure and properties of starch/PVA/nano-SiO hybrid films, Carbohydr. Polym., 2011, 86, 1784-1789. 
7 J. P. Maran, V. Sivakumar, R. Sridhar and V. P. Immanuel, Development of model for mechanical properties of tapioca starch based edible films, Ind. Crops Prod., 2013, 42, 159-168.

8 S. Mali, M. V. E. Grossmann, M. A. García, M. N. Martino and N. E. Zaritzky, Mechanical and thermal properties of yam starch films, Food Hydrocolloids, 2005, 19, 157-164.

9 Y. Y. Zhou, Theory of Textile Warp Sizes, China Textile\& Apparel Press, Beijing, China, 2004, pp. 115-260, (In Chinese).

10 V. J. Davidson, D. Paton, L. L. Diosady and G. J. Larocque, Degradation of wheat starch in a single-screw extruder: characteristics of extruded starch polymers, J. Food Sci., 1984, 49, 453-458.

11 Z. F. Zhu and Z. Q. Cheng, Effect of inorganic phosphates on the adhesion of mono-phosphorylated cornstarch to fibers, Starch/Staerke, 2008, 60, 315-320.

12 Z. F. Zhu and S. J. Cao, Modifications to improve the adhesion of crosslinked starch sizes to fiber substrates, Text. Res. J., 2004, 74, 253-258.

13 Z. F. Zhu, Z. J. Liu, M. L. Li, D. S. Xu and C. L. Li, Monophosphorylation of cornstarch to improve the properties of wool yarns sized at reduced temperature, J. Appl. Polym. Sci., 2013, 127, 127-135.

$14 \mathrm{~W}$. Li, Z. Z. Xu, Z. Q. Wang and J. Xing, One-step quaternization/hydroxypropylsulfonation to improve paste stability, adhesion and film properties of oxidized starch, Polymers, 2018, 10, 1110.

15 M. W. Meshram, V. V. Patil, S. T. Mhaske and B. N. Thorat, Graft copolymers of starch and its application in textiles, Carbohydr. Polym., 2009, 75, 71-78.

16 S. G. Choi and W. L. Kerr, Effects of chemical modification of wheat starch on molecular mobility as studied by pulsed ${ }^{1} \mathrm{H}$ NMR, LWT-Food Sci. Technol., 2003, 51, 1-8.

17 M. J. John, R. Anandjiwala, K. Oksman and A. P. Mathew, Melt-spun polylactic acid fibers: Effect of cellulose nanowhiskers on processing and properties, J. Appl. Polym. Sci., 2012, 127, 274-281.

18 Z. F. Zhu and M. Wang, Effects of starch maleation and sulfosuccination on the adhesion of starch to cotton and polyester fibers, J. Adhes. Sci. Technol., 2014, 28, 935-949.

$19 \mathrm{~J}$. J. Bikerman and D. W. Marshall, Adhesiveness of polyethylene mixture, J. Appl. Polym. Sci., 1963, 7, 1031-1040.

20 L. A. Bello-Pérez, C. A. Bello-Flores, M. D. C. Nunez-Santiago, C. P. Coronel-Aguilera and J. Alvarez-Ramirez, Effect of the degree of substitution of octenyl succinic anhydridebanana starch on emulsion stability, Carbohydr. Polym., 2015, 132, 17-24.

21 Z. F. Zhu and Y. Lei, Effect of chain length of the alkyl in quaternary ammonium substituents on the adhesion-tofiber, aerobic biodegradation, and desizability of quaternized cornstarch, J. Adhes. Sci. Technol., 2015, 29, 116-132.

22 Z. F. Zhu and P. H. Chen, Carbamoyl ethylation of starch for enhancing the adhesion capacity to fibers, J. Appl. Polym. Sci., 2007, 106, 2763-2768.
23 S. Bismark and Z. F. Zhu, Amphipathic starch with phosphate and octenylsuccinate substituents for strong adhesion to cotton in warp sizing, Fibers Polym., 2018, 19, 1850-1860.

$24 \mathrm{~W}$. Li and Z. F. Zhu, Electroneutral maize starch by quaterization and sulfosuccination for strong adhesion-toviscose fibers and easy removal, J. Adhes., 2016, 92, 257-272.

25 W. Li, J. Wu, X. G. Cheng, L. J. Wu, Z. Liu, Q. Q. Ni and Y. H. Lu, Hydroxypropylsulfonation/caproylation of corn starch to enhance its adhesion to PLA fibers for PLA sizing, Polymers, 2019, 11, 1197.

26 W. Li, Z. Z. Xu, Z. Q. Wang, X. H. Liu, C. L. Li and F. T. Ruan, Double etherification of corn starch to improve its adhesion to cotton and polyester fibers, Int. J. Adhes. Adhes., 2018, 84, 101-107.

27 S. A. S. Craig, C. C. Maningat, P. A. Seib and R. C. Hoseney, Starch paste clarity, Cereal Chem., 1989, 66, 173-182.

28 K. S. Sandhu, N. Singh and S. T. Lim, A comparison of native and acid thinned normal andwaxy corn starches: Physicochemical, thermal, morphological andpasting properties, LWT-Food Sci. Technol., 2007, 40, 1527-1536.

29 N. D. S. M. Sechi and P. T. Marques, Preparation and physicochemical, structural and morphological characterization of phosphorylated starch, Mater. Res., 2017, 20, 174-180.

30 J. X. Xie, Infrared spectrum, Science Press, Beijing, 1987.

31 J. Singh, L. Kaur and O. J. Mccarthy, Factors influencing the physico-chemical, morphological, thermal and rheological properties of some chemically modified starches for food applications-A review, Food Hydrocolloids, 2007, 21, 1-22.

32 W. Li, W. Z. Xu, A. F. Wei, Z. Z. Xu and C. H. Zhang, Quaternization/maleation of cornstarch to improve its adhesion and film properties for warp sizing, Fibers Polym., 2016, 17, 1589-1597.

33 A. Alishahi, A. Farahnaky, M. Majzoobi and C. L. Blanchard, Physicochemical and textural properties of corn starch gels: Effect of mixing speed and time, Food Hydrocolloids, 2015, 45, 55-62.

34 K. Zhang, Interface Science of Polymers, China Petrochemical Press, Beijing, 1996.

35 S. Wang and L. Copeland, Effect of Acid Hydrolysis on Starch Structure and Functionality: A Review, Crit. Rev. Food Sci. Nutr., 2015, 55, 1081-1097.

36 A. Rindlav-Westling, M. Stading and P. Gatenholm, Crystallinity and morphology in films of starch, amylose and amylopectin blends, Biomacromolecules, 2002, 3, 84-91.

37 Z. Liu and J. H. Han, Film-forming characterstics of starches, J. Food Sci., 2005, 70, E31-E36.

38 S. H. Wu, Polymer interface and adhesion, Marcel Dekker, New York, 1982.

39 S. Q. Shen, Z. F. Zhu and F. D. Liu, Introduction of poly[(2acryloyloxyethyl trimethyl ammonium chloride)-co-(acrylic acid)] branches onto starch for cotton warp sizing, Carbohydr. Polym., 2016, 138, 280-289.

$40 \mathrm{~W}$. Li and Z. F. Zhu, Effect of sulfosuccinylation of corn starch on the adhesion to viscose fibres at lower temperature, Indian J. Fibre Text. Res., 2014, 39, 314-321. 